\title{
Correction to: Cervical and breast cancer screening uptake among women with serious mental illness: a data linkage study
}

Charlotte Woodhead ${ }^{1 \dagger}$, Ruth Cunningham ${ }^{2+}$, Mark Ashworth ${ }^{3}$, Elizabeth Barley ${ }^{4}$, Robert J. Stewart ${ }^{1 *}$ and Max J. Henderson ${ }^{1}$

\section{Correction to: BMC Cancer (2016) 16:819 https://doi.org/10.1186/}

\section{s12885-016-2842-8}

Following publication of the original article [1], the authors notified us of an error in the reported percentages in Table 3.

\section{Author details}

${ }^{1}$ Institute of Psychiatry, Psychology \& Neuroscience, King's College London, London, UK. ${ }^{2}$ Department of Public Health, University of Otago, Wellington, New Zealand. ${ }^{3}$ Department of Primary Care and Public Health Sciences, King's College London, London, UK. ${ }^{4}$ Facility of Nursing and Midwifery, King's, College London, London, UK.

Received: 7 February 2019 Accepted: 7 February 2019

Published online: 15 February 2019

\section{Reference}

1. Woodhead C, et al. Cervical and breast cancer screening uptake among

women with serious mental illness: a data linkage study. BMC Cancer. 2016; 16:819. https://doi.org/10.1186/s12885-016-2842-8.

* Correspondence: robert.stewart@kcl.ac.uk

${ }^{+}$Charlotte Woodhead and Ruth Cunningham contributed equally to this work.

'Institute of Psychiatry, Psychology \& Neuroscience, King's College London, London, UK

Full list of author information is available at the end of the article 
Table 3 Associations between serious mental illness (SMI) status and recent receipt of breast and/or cervical screening overall and by SMI characteristic sub-group

\begin{tabular}{|c|c|c|c|c|c|c|c|c|}
\hline & \multicolumn{4}{|c|}{ Mammography eligible population $(N=26,010)$} & \multicolumn{4}{|c|}{ Cervical smear eligible population $(N=107,947)$} \\
\hline & $\begin{array}{l}\text { Recorded } \\
\text { mammography } \\
\text { in last } 3 \text { years } \\
\mathrm{n}(\%)\end{array}$ & $\begin{array}{l}\text { Unadjusted } \\
\text { OR (95\% Cl) }\end{array}$ & $\begin{array}{l}\text { Adjusted for } \\
\text { socio- } \\
\text { demographics } \\
\text { OR }{ }^{a}(95 \% \mathrm{Cl})\end{array}$ & $\begin{array}{l}\text { Additionally } \\
\text { adjusted for } \\
\text { consultation rate } \\
\mathrm{OR}^{\mathrm{b}}(95 \% \mathrm{Cl})\end{array}$ & $\begin{array}{l}\text { Recorded } \\
\text { cervical smear } \\
\text { in last } 3 / 5 \text { yrs } \\
\text { n }(\%)\end{array}$ & $\begin{array}{l}\text { Unadjusted } \\
\text { OR (95\% Cl) }\end{array}$ & $\begin{array}{l}\text { Adjusted for } \\
\text { socio- } \\
\text { demographics } \\
\mathrm{OR}^{\mathrm{a}}(95 \% \mathrm{Cl})\end{array}$ & $\begin{array}{l}\text { Additionally } \\
\text { adjusted for } \\
\text { consultation rate } \\
\mathrm{OR}^{\mathrm{b}}(95 \% \mathrm{Cl})\end{array}$ \\
\hline Non-SMI & $14205(56.0)$ & 1.00 & 1.00 & 1.00 & $67823(63.7)$ & 1.00 & 1.00 & 1.00 \\
\hline SMI overall & $305(48.8)$ & $\begin{array}{l}0.72(0.61- \\
0.86)^{* * * *}\end{array}$ & $\begin{array}{l}0.69(0.57- \\
0.84)^{* * * *}\end{array}$ & $0.60(0.49-0.73)^{* * *}$ & $848(60.9)$ & $\begin{array}{l}1.16(0.99- \\
1.35)\end{array}$ & $\begin{array}{l}0.72(0.60- \\
0.85)^{* * * *}\end{array}$ & $0.35(0.29-0.42)^{* * *}$ \\
\hline \multicolumn{9}{|l|}{ SMI by diagnosis } \\
\hline Schizophrenia & $136(47.9)$ & $\begin{array}{l}0.64(0.50- \\
0.82)^{* * * *}\end{array}$ & $\begin{array}{l}0.59(0.45- \\
0.78)^{* * * *}\end{array}$ & $0.52(0.40-0.69)^{* * *}$ & $270(57.3)$ & $\begin{array}{l}0.95(0.74- \\
1.22)\end{array}$ & $\begin{array}{l}0.48(0.36- \\
0.63)^{* * * *}\end{array}$ & $0.24(0.18-0.32)^{* * *}$ \\
\hline $\begin{array}{l}\text { Bipolar } \\
\text { affective } \\
\text { disorder }\end{array}$ & $67(50.4)$ & $\begin{array}{l}0.85(0.58- \\
1.26)\end{array}$ & $\begin{array}{l}0.89(0.59- \\
1.35)\end{array}$ & $0.72(0.47-1.10)$ & $231(67.9)$ & $\begin{array}{l}1.94(1.35- \\
2.78)^{* * *}\end{array}$ & $\begin{array}{l}1.23(0.84 \text { - } \\
1.80)\end{array}$ & $0.50(0.33-0.74)^{* *}$ \\
\hline $\begin{array}{l}\text { Other non- } \\
\text { organic } \\
\text { psychoses }\end{array}$ & $34(41.5)$ & $\begin{array}{l}0.54(0.33- \\
0.87)^{*}\end{array}$ & $\begin{array}{l}0.53(0.31- \\
0.90)^{*}\end{array}$ & $0.47(0.27-0.80)^{* *}$ & $153(56.7)$ & $\begin{array}{l}0.82(0.60- \\
1.13)\end{array}$ & $\begin{array}{l}0.57(0.40- \\
0.80)^{* *}\end{array}$ & $0.33(0.22-0.47)^{* * *}$ \\
\hline \multicolumn{9}{|l|}{ Depot injectable } \\
\hline No & $191(53.4)$ & $\begin{array}{l}0.94(0.74- \\
1.20)\end{array}$ & $\begin{array}{l}0.97(0.75- \\
1.26)\end{array}$ & $0.83(0.64-1.09)$ & $524(63.1)$ & $\begin{array}{l}0.32(1.08- \\
1.62)^{* *}\end{array}$ & $\begin{array}{l}0.82(0.65- \\
1.02)\end{array}$ & $0.36(0.29-0.46)^{* * *}$ \\
\hline Yes & $80(42.6)$ & $\begin{array}{l}0.50(0.37- \\
0.68)^{* * *}\end{array}$ & $\begin{array}{l}0.46(0.33- \\
0.64)^{* * * *}\end{array}$ & $0.39(0.27-0.54)^{* * *}$ & $199(58.5)$ & $\begin{array}{l}0.92(0.69- \\
1.22)\end{array}$ & $\begin{array}{l}0.48(0.35- \\
0.66)^{* * * *}\end{array}$ & $0.26(0.18-0.36)^{* * *}$ \\
\hline \multicolumn{9}{|l|}{$\begin{array}{l}\text { Any indicator } \\
\text { of severity }\end{array}$} \\
\hline No & $168(53.7)$ & $\begin{array}{l}1.00(0.77 \text { - } \\
1.29)\end{array}$ & $\begin{array}{l}0.91(0.69- \\
1.21)\end{array}$ & $0.79(0.59-1.06)$ & $411(62.7)$ & $\begin{array}{l}1.32(1.05- \\
1.66)^{*}\end{array}$ & $\begin{array}{l}0.82(0.63- \\
1.06)\end{array}$ & $0.40(0.31-0.53)^{* * *}$ \\
\hline Yes & $137(43.9)$ & $\begin{array}{l}0.54(0.43- \\
0.69)^{* * *}\end{array}$ & $\begin{array}{l}0.54(0.42- \\
0.70)^{* * *}\end{array}$ & $0.46(0.36-0.61)^{* * *}$ & $437(59.2)$ & $\begin{array}{l}1.04(0.85- \\
1.27)\end{array}$ & $\begin{array}{l}0.65(0.52- \\
0.81)^{* * * *}\end{array}$ & $0.31(0.25-0.40)^{* * *}$ \\
\hline \multicolumn{9}{|l|}{$\begin{array}{l}\text { Any indicator } \\
\text { of risk }{ }^{2}\end{array}$} \\
\hline No & $205(51.4)$ & $\begin{array}{l}0.84(0.67- \\
1.05)\end{array}$ & $\begin{array}{l}0.81(0.63- \\
1.03)\end{array}$ & $\begin{array}{l}0.70(0.55- \\
0.90)^{* *}\end{array}$ & $535(62.9)$ & $\begin{array}{l}1.31(1.07- \\
1.60)^{* *}\end{array}$ & $\begin{array}{l}0.79(0.63- \\
1.00)^{*}\end{array}$ & $0.38(0.30-0.49)^{* * *}$ \\
\hline Yes & $100(44.2)$ & $\begin{array}{l}0.56(0.42- \\
0.74)^{* * * *}\end{array}$ & $\begin{array}{l}0.53(0.39- \\
0.73)^{* * *}\end{array}$ & $\begin{array}{l}0.46(0.34- \\
0.63)^{* * * *}\end{array}$ & $313(57.6)$ & $\begin{array}{l}0.97(0.77- \\
1.23)\end{array}$ & $\begin{array}{l}0.62(0.48- \\
0.80)^{* * *}\end{array}$ & $0.31(0.24-0.41)^{* * *}$ \\
\hline
\end{tabular}

Eligible population includes non-linked non-SMI and linked SMI group

${ }^{*} p<0.05,{ }^{* *} p<0.01,{ }^{* * *} p<0.001$

${ }^{1}$ Includes any of: ever had an inpatient stay, any record of being treated under the Mental Health Act, any record of difficulty managing their physical health, or any record of an Assertive Outreach/Crisis/A\&E episode

${ }^{2}$ Includes any of: recorded history of violence, recorded history of non-compliance, and any record of a forensic history

a Adjusted for age (continuous), ethnicity, and borough-level deprivation

${ }^{\mathrm{b}}$ Additionally adjusted for mean annual number of primary consultations 